\title{
REMARK ON THE ZERO LOCUS OF A SMOOTH FUNCTION
}

\author{
D. BURNS, JR. ${ }^{1}$
}

\begin{abstract}
A sufficient condition is given to insure the existence of some smooth points in the zero set of a $C^{\infty}$ function.
\end{abstract}

Since any closed set in $\mathbf{R}^{n}$ is the zero locus of a $C^{\infty}$ function, there is little to be said, in general, about such a set. Even if the function $f$ vanishes to finite order at the origin, i.e., $D^{\alpha} f(0) \neq 0$ for some $\alpha$, the zero set $Z$ of $f$ near 0 still need not have much geometric structure.

ExAmple. Let $Y \subset \mathbf{R}$ be a Cantor set, and let $\varphi(y)$ be a $C^{\infty}$ function on $\mathbf{R}$, nonnegative, zero only along $Y . f(x, y)=x^{2}+\varphi(y)$ is zero only along $\{(0, y) \mid y \in Y\}$.

However, if the zero locus of an $f$ vanishing to finite order is in some sense a boundary between two regions, we can say more.

THEOREM. Let $f \in C^{\infty}, f(0)=0 ; D^{\alpha} f(0) \neq 0$, some $\alpha$. Suppose $0 \in \bar{\Omega}^{+} \cap$ $\vec{\Omega}^{-}$, where $\Omega^{ \pm}=\{x \mid \pm f(x)>0\}$. Then there is an open subset $\theta$ of $Z$ where, for each $p \in \mathcal{O}$, there is a neighborhood $U$ of $p$ in $\mathbf{R}^{n}$ such that $U \cap Z$ is a $C^{\infty}$ submanifold of $R^{n}$, and $0 \in \overline{\mathcal{O}}$.

Proof. Malgrange's preparation theorem says there exist coordinates $x=$ $\left(x_{1}, \ldots, x_{n-1}, x_{n}\right)=\left(x^{\prime}, x_{n}\right)$ at 0 such that

$$
f(x)=h(x)\left(x_{n}^{k}+a_{k-1}\left(x^{\prime}\right) x_{n}^{k-1}+\cdots+a_{0}\left(x^{\prime}\right)\right)=h(x) P_{x^{\prime}}\left(x_{n}\right)
$$

with $h$ and the $a_{i} \in C^{\infty}, h(0) \neq 0$. Let $\pi$ denote the projection from $\mathbf{R}^{n} \rightarrow$ $\mathbf{R}^{n-1},\left(x^{\prime}, x_{n}\right) \rightarrow x^{\prime}$, and set $\omega^{ \pm}=\pi\left(\Omega^{ \pm}\right) ; \omega^{ \pm}$are open in $\mathbf{R}^{n-1}$. Take any neighborhood $V \times(-\varepsilon, \varepsilon)$ about 0 , where $\varepsilon>0$ and $V$ is connected and open in $\mathbf{R}^{n-1}$. We first note that $\omega^{+} \cap \omega^{-} \neq \varnothing$ in $V$ : otherwise, $\exists x^{\prime}$ such that $\pi^{-1}\left(x^{\prime}\right) \cap\left(\Omega^{+} \cup \Omega^{-}\right)=\varnothing$, i.e., $P_{x^{\prime}}\left(x_{n}\right)$ is identically zero, a contradiction. Now $P_{x^{\prime}}\left(x_{n}\right)$ has zeroes over every point of $\omega^{+} \cap \omega^{-}$, and we have only to isolate branches of these zeroes.

For $x^{\prime} \in \omega^{+} \cap \omega^{-}$, let $N\left(x^{\prime}\right)=$ the number of odd zeroes of $P_{x^{\prime}}\left(x_{n}\right)=$ the number of roots $\xi$ of $P_{x^{\prime}}\left(x_{n}\right)=0$ with odd multiplicity $N\left(x^{\prime}\right)$ is integer-valued, lower-semicontinuous, positive and bounded by $k$ on $\omega^{+} \cap \omega^{-}$. Let $\omega \subset \omega^{+}$ $\cap \omega^{-}$be the open subset where $N\left(x^{\prime}\right)$ assumes its maximum on $\omega^{+} \cap \omega^{-}$. Next, consider for $x^{\prime} \in \omega^{+} \cap \omega^{-}$,

Received by the editors September 12, 1977.

AMS (MOS) subject classifications (1970). Primary 58A05.

Key words and phrases. Zero locus, smooth function.

${ }^{1}$ Partially supported by N.S.F. 


$$
M\left(x^{\prime}\right)=\sum \text { multiplicity }(\xi),
$$

the summation taken over all odd roots $\xi$ of $P_{x^{\prime}}\left(x_{n}\right)=0$. It is easy to see that $M\left(x^{\prime}\right)$ is integer-valued, upper-semicontinuous, positive and bounded on $\omega$. Let $\omega^{\prime}$ be the open subset of $\omega$ where $M\left(x^{\prime}\right)$ assumes its minimum value on $\omega$.

For $x^{\prime} \in \omega^{\prime}$, let $\xi^{\prime}$ be an odd zero of $P_{x^{\prime}}\left(x_{n}\right)$. Then, given $\varepsilon>0$, if $\left|x^{\prime \prime}-x^{\prime}\right|<\delta, x^{\prime \prime} \in \mathbf{R}^{n-1}$ and $\delta$ small enough, $N\left(x^{\prime}\right)=N\left(x^{\prime \prime}\right)$ and there is a unique odd zero $\xi^{\prime \prime}$ of $P_{x^{\prime \prime}}\left(x_{n}\right)$ with $\left|\xi^{\prime}-\xi^{\prime \prime}\right|<\varepsilon$, and we have multiplicity $\left(\xi^{\prime \prime}\right)$ < multiplicity $\left(\xi^{\prime}\right)$. If $\delta$ is small enough, however, $M\left(x^{\prime \prime}\right)=M\left(x^{\prime}\right)$, and

$$
d=\operatorname{multiplicity}\left(\xi^{\prime \prime}\right)=\operatorname{multiplicity}\left(\xi^{\prime}\right)
$$

and $\xi^{\prime \prime}$ is the unique zero of $P_{x^{\prime \prime}}\left(x_{n}\right)$ within $\varepsilon$ of $\xi^{\prime}$. Thus, in the cylinder

$$
U=\left\{\left(y^{\prime}, y_{n}\right)|| y^{\prime}-x^{\prime}|<\delta,| x_{n}-y_{n} \mid<\varepsilon\right\},
$$

the set

$$
Z=\left\{\left(x^{\prime}, x_{n}\right) \mid f\left(x^{\prime}, x_{n}\right)=0\right\}=\left\{\left(x^{\prime}, x_{n}\right) \mid P_{x^{\prime}}\left(x_{n}\right)=0\right\}
$$

is identical with the set $\left\{\left(x^{\prime}, x_{n}\right) \mid\left(\partial^{d-1} / \partial x_{n}^{d-1}\right) P_{x^{\prime}}\left(x_{n}\right)=0\right\}$, which is a smooth submanifold of $U$.

It seems unlikely that a more precise statement than the above can be made, e.g., about the measure-theoretic size of the set $\mathcal{O}$. Its Hausdorff 1-dimensional density at 0 , for example, can be made arbitrarily small. It is easy to see that the hypothesis $D^{\alpha} f(0) \neq 0$ cannot be dropped from the statement of the theorem.

The author thanks $\mathbf{J}$. Mather for suggesting the example given above.

Department of Mathematics, Princeton University, Princeton, New Jersey 08540 